Research Journal of Applied Sciences 7 (2): 71-83, 2012

ISSN: $1815-932 \mathrm{X}$

(C) Medwell Journals, 2012

\title{
Development of a Routing Framework for a Cluster-Based Congestion Avoidance and Load Balancing Algorithm for IEEE802.11s Mesh Network
}

\author{
Adebanjo Adekiigbe and Kamalrulnizam Abu Bakar \\ Department of Computer Systems and Communication, \\ Faculty of Computer Science and Information Systems, Universiti Teknologi Malaysia, \\ Kuala Lumpur, Malaysia
}

\begin{abstract}
This study is focused on the design and development of efficient congestion avoidance and routing algorithm framework in Wireless Mesh Networks (WMNs). The framework will employ the use of clustering algorithm to booster the routing protocol with particular attention being paid to the aggregated data packets at the intermediate nodes toward the gateway in wireless mesh network. The design uses the enhanced weighted clustering algorithm as base algorithm because of the advantages identified in this protocol from previous research. Efforts are made to integrate congestion avoidance, load balancing routing and the clustering algorithm with the greater hope of achieving low routing overheads, end to end delay while throughout and data delivery ratio expected to witness high increase.
\end{abstract}

Key words: Routing, clustering, congestion avoidance, load balancing, overheads, throughput, delay

\section{INTRODUCTION}

A Wireless Mesh Network (WMN) is a network made up of nodes organized in a mesh topology. Mesh topology is a type of layout pattern of interconnections of the various nodes where each node must not only capture and disseminate its own data but also serve as a connector to another node in the network for purpose of collaboration to propagate the data packet in the network thus, a mesh network adjudged to be reliable and offers redundancy (Akyildiz and Wang, 2009).

The compositions of WMN are mesh clients, mesh routers and gateways. The mesh clients are those facilities that are connected to the network for the purpose of receiving or sending data from other facilities. Such facilities are laptops, cell phones and other wireless devices while the mesh routers forward traffic to and from the gateways which may either be connected to the Internet or wired LAN. If a member node can no longer operate due to link or power failure, the rest of the nodes can still communicate with each other, directly or through one or more intermediate nodes. Since, it offers reliability and redundancy, it can be deployed for network connectivity in areas where it is topologically and technically difficult to lay cables for wired connection for the provisioning of dynamic and cost effective connectivity. Unlike the ad hoc networks, WMNs are not most often resource constraint hence, it can be subjected to performing resource demanding functions such as congestion control or routing of traffic data in this case infrastructure WMNs are the reference point. Whereas the hybrid WMN can experience resource constraints if the hybridization involves wireless sensor networks (Methley, 2009).

Wireless mesh architectures infrastructure is in effect, a router network which is built with peer radio devices without need to use cable between different nodes as is the practice in the traditional Wireless Local Area Network (WLAN) Access Points (APs). Mesh structural design sustains signal strength by breaking long distances into a series of shorter hops. Intermediate nodes not only boost the signal but supportively make forwarding decisions based on their knowledge of the network that is perform routing and other data traffic essential tasks. Such an architecture if carefully designed may provide high bandwidth, spectral efficiency and economic advantage over the coverage area (Jun and Sichitiu, 2003; Zimmermann et al., 2007). Figure 1 shows a schematic diagram of a hypothetical WMN which comprises of mesh clients, mesh routers and gateways.

Due to its fast deployment, easy maintenance and low upfront investments, WMN has gained considerable attention in recent years. Moreover, it is reliable and offers redundancy. When there is a link or node failure, the nodes can still communicate with each other by using other intermediate nodes hence, WMN is dynamically

Corresponding Author: Adebanjo Adekiigbe, Department of Computer Systems and Communication, Faculty of Computer Science and Information Systems, Universiti Teknologi Malaysia, Kuala Lumpur, Malaysia 


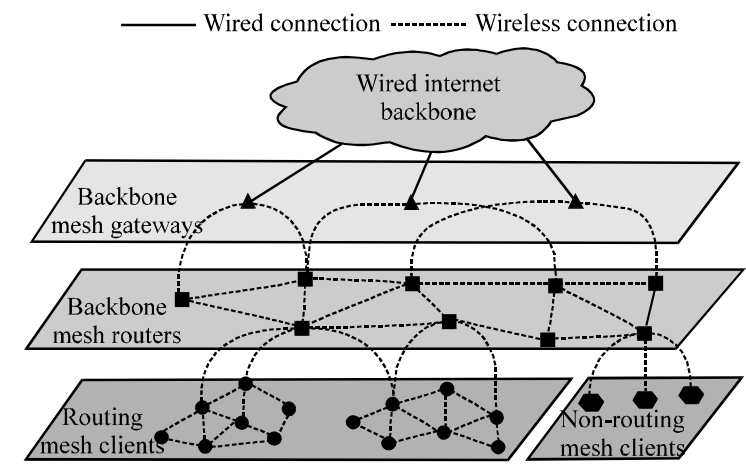

Fig. 1: Wireless mesh network topology

self-organized and self-configured. It has a number of features that distinguish it from pure ad hoc networks. First, the positions of different nodes of a $\mathrm{WMN}$ are relatively fixed (quasi-static) that is any change of position is limited within certain range. Secondly, unlike pure ad hoc networks where traffic flows between arbitrary pairs of nodes in $\mathrm{WMN}$, all traffic flows are either to or from a designated gateway which connects the WMN to the internet or wired Local Area Networks (LAN) and thirdly, mesh routing protocols do not have energy consumption restrictions since, mesh routers should most likely be on wired power (Raniwala and Chiueh, 2005).

In view of the wide deployment of WMNs, a critical challenge facing this network is the design and development of protocols that can simultaneously guarantee quality of service and high bandwidth utilization, high throughput, low delay for delay sensitive applications such as real time applications. Each node tries to send as much data as possible over the link, congestion or overflow between mesh nodes can occur and hence, overall network performance can degrade (Yi and Shakkottai, 2007; Akyildiz et al., 2005).

\section{MOTIVATION AND PROBLEM BACKGROUND}

Wireless Mesh Networks (WMNs) have a wide range of applications in Mobile $A d$ hoc (MANET), Vehicular $A d$ hoc Network (VANET), Wireless Sensor Network (WSN), broadband home networking, community and neighborhood networking, enterprise networking, metropolitan, building, transportation systems, emergency/disaster (spontaneous) networks (Akyildiz and Wang, 2009). WMNs differ from traditional wireless networks in many aspects (Akyildiz et al., 2005). For instance, the sending rate of a mobile node is not only determined by the channel capacity but also by the

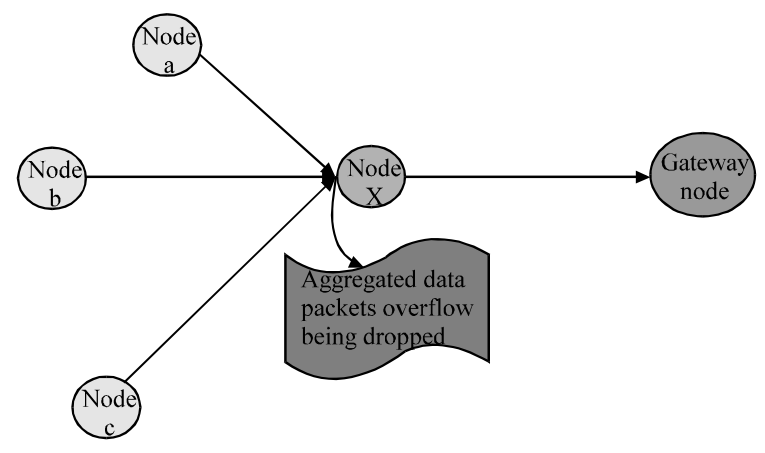

Fig. 2: CSMA causes buffer overflow

activity of the neighboring mobile nodes. Whenever a mobile node receives more data than it can forward, the excess data has to be buffered. Congestion occurs when the limited buffer space is full and consequently the received data has to be dropped. The dropped packets will either be retransmitted or totally forgotten this will cause the throughput to be highly degraded, especially when Real Time Applications (RTAs) are to be supported. Real time traffics require instantaneous response from both the sender and the receiver.

As an illustration of the scenario of buffer overflow, Fig. 2 shows an intermediate mobile node $\mathrm{x}$ that is close to the gateway node is likely to have multiple upstream mobile nodes such as nodes a-c. With CSMA, the upstream mobile nodes jointly have more chance to forward packets to $\mathrm{x}$ than $\mathrm{x}$ can send out. The excessive packets received by $\mathrm{x}$ will eventually cause buffer overflow. Consequently, hotspots may form around the bottleneck mobile nodes. Hence, buffer overflow in the intermediate mobile node (that is node $\mathrm{x}$ ) amount to waste of wireless bandwidth that was used in transmitting the data packets from the source node to its present state. Apparently, the mesh network performance degrades with low throughput due to data packet drop, delay and additional overheads are incurred for retransmission, these are some of the problems of congestion (Welzl, 2005). The more the data packets advanced towards the gateway node, the more the resources are being wasted if the packets are eventually dropped.

Whenever the buffer at a node $\mathrm{x}$ is full if the upstream neighbors attempt to send data packets to $\mathrm{x}$, their efforts are supposedly turn to a wasted one and worse still, counter-productive if for example, their RTS packets collide with nearby transmissions, it will cause throughput reduction of other nodes. At last and above all, the data loss due to congestion will endanger the mission of the real time applications. 


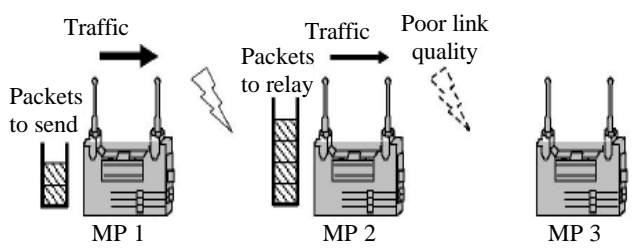

Fig. 3: Buffer overflow issue due to poor link (Yi and Shakkottai, 2007)

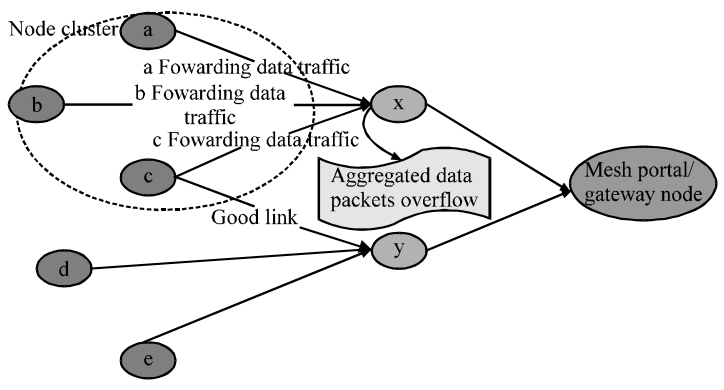

Fig. 4: Unbalanced traffic load between node $\mathrm{x}$ and $\mathrm{y}$

In a related development, the scenario shown in Fig. 3 shows that buffer overflow can also arise owing to variance in link qualities which results in different transmission rates. Suppose a traffic flow originates from mesh point 1 which has mesh point 3 as its destination node, definitely the traffic flow traverses through mesh point 2 . If the link quality between mesh point 1 and 2 is of healthier quality than what existed between mesh point 2 and 3 as a result the transmission rate of mesh point 1 will be more than the transmission rate of mesh point 2 since, mesh point 2 will continually experience retransmission of dropped packets caused by poor link path with its one-hop neighbor. Therefore, if mesh points 1 and 2 have equal number of opportunities to access channel, mesh point 2 cannot transmit all data packets received from mesh point 1 because of its low transmission rate. Hence, buffer overflow in the intermediate mesh point (that is mesh point 2) amount to waste of wireless bandwidth that was used in transmitting the data packets from the source node to its present state. Apparently, the mesh network performance degrades with low throughput due to data packet drop, delay and additional overheads are incurred for retransmission.

Issues bothering on load balancing were also of main concern to realization of the design objectives of $\mathrm{WMNs}$, especially during the pick period of the network usage. Figure 4 shows a case of unbalanced load traffic. If traffic loads being transmitted from node $\mathrm{c}$ is forwarded to node $\mathrm{x}$, the chance is that node $\mathrm{x}$ may become bottleneck since node $\mathrm{a}$ and $\mathrm{b}$ are already forwarding to node $\mathrm{x}$.
Since, good link exist between nodes c and $y$, the node $\mathrm{c}$ data traffic could have been transmitted to the gateway through node $y$ which is idle as at the time of this transmission if a load balancing technique is used and this would have saved node $\mathrm{x}$ probably from being bottlenecked.

The main concern is whether the performance of WMNs congestion control and routing protocols can satisfy the throughput and delay requirements of real time applications in a community networking particularly in urban city daytime scenarios where business offices are densely packed and network usage is at pick. Therefore, according to the issues raised, the designing of congestion avoidance protocol with a load balancing mechanisms to satisfy performance demands of the various RTAs in a community mesh network is required and considered as an important research area as many of these performance parameters should be satisfied during data transmission from the mesh source node to the destination node.

The IEEE 802.11 s was designed based on IEEE802.11e MAC that made provision for differentiated services through the adjustment of access parameters to guarantee Quality of Service (QoS) performance demand requirements of various applications (Fujiwara et al., 2006; Choi et al., 2003) however, the challenges before IEEE 802.11s MAC protocol is that source nodes from different area of the network sends data traffic as much as possible across the link to destination nodes but the capability and traffic load capacity for each individual link over a multiple hop path may vary significantly ( $\mathrm{Yi}$ and Shakkottai, 2007).

Traditional routing protocols such as Routing Information Protocol (RIP) (Hendrik, 1988) cannot be used in WMNs to route real time traffics due to their peculiar characteristics which resemble that of mobile ad hoc networks with dynamic mobility, frequent disconnections, low bandwidth, low battery power, etc. These characteristics make the routing a daunting task in WMNs. The routing technique used for packets forwarding will either make or mar the successful delivery with additional overheads being incurred by the network, from the source to destination nodes. Even though routing in wireless networks has gone through farreaching study, $A d$ hoc On-Demand Distance Vector (AODV) (Perkins et al., 2003), Dynamic Source Routing (DSR) (Johnson and Maltz, 1996) and Optimized Link State Routing (OLSR) (Clausen and Jacquet, 2003) which are designed for mobile ad hoc networks MANET are mostly used routing protocols in Wireless Mesh Network (WMN) (Mugaibel and Othman, 2009; Rahman et al., 2009). OLSR is a proactive routing protocol that made every node in the network to maintain a routing table to 
store information so that the routes are always available when needed, nevertheless, the routing overheads increases when each node periodically sends the topology information all through the whole network this has made it not very useful in a large scale network like WMNs. Whereas the reactive routing protocols such as AODV and DRS tend to reduce the routing overheads but increased the delay due to the finding of new routes by invoking route discovery through flooding mechanism when the source node wants to establish a route to the destination. Proactive routing has been adjudged to be better than reactive routing for stationary network while reactive is better for highly mobile network (Kum et al., 2010).

The AODV and DSR routing protocols have been established to perform poorly in the WMNs (Lee and Ward, 2004; Nandiraju et al., 2006) because the traffic model of WMNs aggregate data packets towards or away from the default gateway nodes which may lead to subdued performance due to the presence of hotspots close to the default gateway node. Neither AODV nor DSR were designed to include efficient load balancing capabilities that would ensure that a node does not become a bottleneck. AODV and DSR are also generating high routing and control information overheads which increase as the network scale larger. To address some of these problems with AODV and DSR for IEEE802.11s routing protocols, researchers have suggested various exploits of achieving a routing that will reduce congestions in WMNs. Specifically, hierarchical routing or cluster based routing protocols has be seen to improve the routing overheads and reduced congestions (Jiang et al., 1999).

\section{REVIEW OF RELATED PROPOSALS}

In a cluster based routing, data traffics are routes from source node to destination node using a hierarchical cluster heads. This simple act is bound to reduce the routing overheads for the networks. Jiang et al. (1999) introduced Cluster-Based Routing Protocols (CBRP) to reduce computational overheads, Wan et al. (2008) summarized the advantages of cluster routing as follows:

- Reduction of flooding overheads devoid of network performance degradation during route path construction stage

- Aggregation of data within a cluster reduces messages that flow during data-transmitting process

- Node mobility that may require the update of the entire network can be easily handled within a cluster where the node moves during the route maintenance process
Many proposals have been presented on cluster based routing protocol with its attendants advantages over the flat structure. However, most of these protocols still lag in supporting the WMNs to balance load and save the network from congestions at bottlenecked nodes.

In CBRP (Jiang et al., 1999) the clustering process divides all nodes into clusters with a clustering algorithm. A variation of the lowest-ID clustering algorithm proposed by Gerla and Tsai (1995), identifier-based algorithm is used for CBRP as a base algorithm. The algorithm ensured that when a node comes up, it enters the undecided state, starting its timer and ensured that a hello message is broadcasted. The cluster head responded whenever it got the hello messages by triggering the hello message immediately. The limitation of CBRP becomes glaring when a cluster or the network is too big the overhead on each packet grows because of source routing since, every node in routing path has to be saved in the routed packet. Hence, the packet size increases relative to the path length of the route; this as well increases transmission time. Another difficulty of the CBRP is in its support for links that are uni-directional when using a network with 802.11 link layer technology these links cannot be supported because the 802.11 protocol only understands links that are bi-directional. Based on the view of the 802.11 protocol, one node would have to be permitted to forward acknowledgement packets so that, an acknowledgement would have to be sent back to sender by a node with the use of multiple hops. Again this routing technique does not give consideration to the traffic pattern of the WMNs. No attempt is made to control congestions at the bottleneck nodes. CBRP routing is done using source routing, the problems with DSR such as higher connection setup delay and high routing overheads cannot also be isolated with CBRP.

CGSR (Liu et al., 1997) uses DSDV as an underlying protocol, network nodes are partition into clusters while distributed algorithm is used to select the cluster head. Any node that is within the communication range of a particular cluster head is a member of that cluster whereas a node that falls within the communication range of two or more cluster heads is referred to as gateway node. The clustering process of CGSR adopts Least Cluster Change (LCC) clustering algorithm where a cluster head change occurs whenever two cluster heads move toward into one cluster or one of the nodes moves out of the cluster heads communication range. The cluster head controls all broadcasting in a cluster, forwards messages on behalf of member nodes and perform dynamic channel scheduling. All nodes in the cluster maintains two tables that is a table that contains cluster member and the cluster head for each 
destination node, a distance vector routing table which contains the next hop to the destination. A periodical broadcast of the cluster member table is done which allow a node to update its entries in the cluster member table whenever it receives a new one from its neighbors. Like DSDV it also, uses sequence numbers. In CGRS, node routes packets by finding the nearest cluster head along the path to destination based on the routing table and cluster member table, it then transmit the packet to the node after consultation with routing table for the next hop to reach the cluster head selected beforehand. In the first instance, the packet source node will transmit the packet to its cluster head while the cluster head forwards the packet to the cluster gateway node which connects the cluster head with the next cluster head along the path to the destination. The cluster gateway forwards the packet to the next cluster head this process continues until the packet is delivered to the destination cluster head while the cluster head at destination cluster transmit the packet to the destination node.

Routing in CSGR is more effective than DSDV because it is done through the cluster heads and cluster gateway nodes. However, the routing was not designed to consider bottleneck nodes towards the default gateway node. The traffic pattern in WMNs put more responsibility on the nodes close to the default gateway due to aggregation of data packets coming from different zones of the network. For real time traffic, the minimum delay experienced from source nodes to the point of bottleneck would have been eroded due to queue up of packets at the bottleneck nodes and that the packets transfer would eventually be dropped if the queue continue to grow and the network throughput will be seriously degraded, therefore QoS requirements of the RTAs is reduced. Another implication of the protocol is that no adequate control is placed on the cluster head to sort out congestions at its level. It is very important to control congestions at the cluster heads as all member nodes may wish to transmit data packets to it at the same time. The cluster head is saddled with higher computation and communication load than other nodes hence, the traffic bottleneck and single point failures at the cluster heads and gateways will results in path length increases and high overheads. The performance is degraded by the following facts also, the selection of the cluster heads may cause complexity and overhead while this become more difficult to the cluster structure in mobile environment.

Nandiraju et al. (2006) proposed a load balancing scheme which is congestion aware. The proposal has two functional phases: the first phase is a protocol for gateway discovery while the second phase was for load migration. The gateways announce itself by sending beacons periodically to all its intermediate nodes and all nodes that have not selected a gateway node is registered to its nearest gateway node or if the present gateway node is closer to a node than the previous node its registered with it will change its gateway node to the new one closer. Such, a situation prompts the node to store the originally registered longer hop gateway as its secondary gateway which can be exploited to balance load within the network. The load migration procedure exploit the mesh routers by creating gateway table which consist of GW IDs this information is gotten through periodic HELLO packets from intermediate nodes. Following the preliminary gateway discovery procedure, the load migration provides the scheme that allow the IGW to always monitors its queue length during a time window, any queue length above a chosen threshold between a time period signals imminent congestion at IGW then IGW identifies sources with high traffic and send notification that carries message such as queue length average and the exceeded capacity which will be sent unicast messages because the probability of loss of packets is to a great extent reduced, again if the CONGEST_NOTIFY message is broadcasted, all the nodes that are routing through IGW that sends congestion notification will switch to a another IGW which results in load on the sender of congestion notification be highly reduced while new IGW will be highly overloaded. The admittance of the node to the gateway is subject to the queue length and number of flows if the node is accepted, it sends Gateway Reply (GW_REP) to the node that to be serviced and the node switches to the new alternate gateway. The proposal showed that packet delivery ratio of the flows in the load balancing schee is higher than without load balancing because congestion builds up at the gateway resulting in increased packet loss, it was noticed that average delay in the network for the flows with load balancing scheme is lesser than the default scheme because different gateways shared the traffic load which make IGW to experienced less congestion. However, the routing overheads was not seems to have been minimized due to notification messages that needed to be sent to sources nodes from the gateway node. This is an additional burden for a large network like WMNs and this will increase the end to end delay for real time traffics.

Schoeneich and Golanski (2007) presented a proposal called Mesh Cluster Based Routing Protocol (MCBRP) which grouped wireless nodes into clusters where clusterhead are also saddled with the responsible of communication management. The major concern about the proposal was extending the range limitation of many 
number of access points to be able to cover large areas especially when users internet connections in areas that does not have fixed wireless infrastructure. In other to extend the available transmission range, all nodes must act as a router. The protocol ensures that all cluster members have access to the internet this requires all nodes to be part of routing process while a mechanism of automatic forwarding of data from a Cluster Hard-Head $(\mathrm{CHH})$ to another $\mathrm{CHH}$ despite a link breakage to the Internet is required. The proposal modifies CBRP to achieve this with some basic assumptions that all nodes must work in ad hoc mode, many number of CHH's is needed to be used, $\mathrm{CM}$ and $\mathrm{CHH}$ requires communication principles between them, every $\mathrm{CHH}$ should be able to implement the ability to be a gateway node and Duplication Address Detection (DAD) algorithm must be used for address assignment. The researchers claimed that the implementation works properly and that data transmission delays were not significant. They further claimed that the protocol function well even with bigger networks. Despite the proposal lofty idea of scaling the network transmission range efforts are not made to tame buffer overflow where data traffics are aggregated at the mesh node connection with the internet neither did any mechanism established load balancing for the system.

Attempt made by Dang and Wu (2008) was to reduce the overheads of the routing protocol by avoiding cluster head selection where cluster gateway nodes take up the responsibility of cluster heads. The protocol allows source node $\mathrm{x}$ to transmit to destination node $\mathrm{y}$ in the same cluster provided they can link up with each other using intra cluster routing. However if transmitting and the receiving nodes are not in the same cluster, node $\mathrm{x}$ looks up for gateway information to node y's cluster within its gateway table. Node $\mathrm{x}$ transmit data message to the gateway node if an entry is found in its table. Whenever the gateway node received the data message, it forwarded it to any node in the node y cluster, the receiving node in turn deliver it to node $\mathrm{y}$, respectively through intra cluster routing.

Multi-hop inter cluster routing is used whenever the source node $\mathrm{x}$ cannot locate gateway entry information about the destination node $y$. The multi hop inter cluster routing scheme work by employing link state like protocol because in a low connectivity environment on demand routing protocol will work ineffectively with probability of high packet dropping. The researchers built the multi hop inter cluster routing scheme by ensuring that all gateway node constructs a Cluster Connectivity Packet (CCP) and broadcasts it to all other gateway nodes in the network. The gateway CCP is made up of its cluster ID and a list of clusters to which it serves as gateway alongside the matching contact probabilities. Network graph is constructed the moment a gateway node accumulates a sufficient CCP's set. The vertex in the graph represents a cluster. A link connects two vertices if there is a gateway between these two clusters. The weight of the link is the contact probability of the corresponding gateway nodes. The shortest path algorithm is employed to establish the routing table because of the network graph, the routing table contain destination cluster ID and the cluster ID of next hop cluster. The proposal was enhanced with a load balancing to share traffic among the nodes within the same cluster. The node performs load balancing by transmitting at random as many packets as possible to any other node it meets when queues in the node exceeds a pre-defined threshold, until their queues are of the same length. The protocol was simulated using a standalone simulator which adopts the Community-Based Mobility Model (Spyropoulos et al., 2006). The researchers claimed the simulation results show that protocol achieves higher delivery ratio and significantly lower overhead and end to end delay in most simulation scenarios when compared with its non clustering routing protocols. However, aggregated data at the bottleneck towards the default gateway node was not taken care of. The performance metrics presented in this research cannot be said to be better than some previous studies that uses cluster based routing approach because the research was not compared with any existing cluster based routing protocols since, the performance analysis was done with flat network routing protocols. In another research proposed by Dang and Wu (2010), the emphasis was on a delay tolerant mobile node in the study, they investigated distributed clustering and cluster-based routing protocols for Delay-Tolerant Mobile Networks (DTMNs) Grouping mobile nodes with similar mobility pattern into the same cluster where nodes will separately learn unknown and probably random mobility parameters where the nodes in a cluster can then share their resources to reduce overhead and balancing load balancing interchangeably. This ideal is aimed at achieving scalable and efficient routing. Nevertheless, the routing does not accord buffer overflow in at a bottleneck node in the network any priority. This makes the work not to be applicable to delay sensitive applications like RTAs. The main goal of the DTH-based Cluster Routing Protocol (DCRP) proposed by Pinheiro et al. (2009) was to achieve high scalability in topologies where the mobility of the mesh point is low or even zero in which case, a scalable network will tolerate fault and reduce congestion conditions. The protocol proposed path selection using distributed hash tables and RA-OLSR. DCRP partitions a WMN into clusters with each cluster having its own identity. The MPs in a cluster 
execute an intra-cluster routing protocol to exchange routing information for the routes to MPs within the cluster. The intra-cluster routing help reduce the total amount of routing information exchanged since all other information for other MPs in other clusters are not included. The reduction in information exchange is more effective in topologies where the MPs are mostly immobile and mesh clients are highly mobile by frequently changing the Mesh Access Point (MAP). In other to support communication among nodes in different clusters the DCRP uses an inter-cluster routing protocol. The protocol is executed only by the MPs of the cluster that are connected to MPs of other clusters. These MPs are called cluster's boarder MPs while MPs in a cluster that are connected only to MPs of the same clusters are called internal MPs.

A DCRP uses centralize clustering algorithm approach though, simple and is likely to lead to better clustering decisions because they are based on complete knowledge of the network topology. However, this algorithm seems impracticable in real world situation where a number of nodes may not be known a priori, scalability will become a major issue in such a large network of nodes. A distributive approach is may be better though will impose more overheads.

Shahverdy et al. (2011) presented research on the load balancing at the intermediate nodes to the gateway. They argued that previous load balancing algorithms as was presented in Manoj and Rao (2006) does not spot fast traffic ripple that is when the numbers of nodes transferring towards the gateway node suddenly increases this force an increase of work load on the router and transmission nodes. The problem was said to decrease network performance due to aggregated data packets at the intermediate transmission nodes which have been prevented to get to the destination node on time. The researchers used clustering techniques to group nodes that are within the transmission range of each other for better control of workload on nodes while a cluster gateway was selected for each cluster. The algorithm in this proposal works by having two states for a node trying to send data to a destination node: in the first instance when the destination node is within the same cluster as source node, transmission is done with one hop while the second instance requires that the data be sent to cluster gateway node of its cluster and thereafter forward to the destination nodes.

The clustering approach is not too different from the research presented by Dang and Wu (2008). However, the basic ideal presented was the breaking of the cluster into two halves to resolve congestion in the cluster gateway when it is expedient that numbers of nodes sending traffic

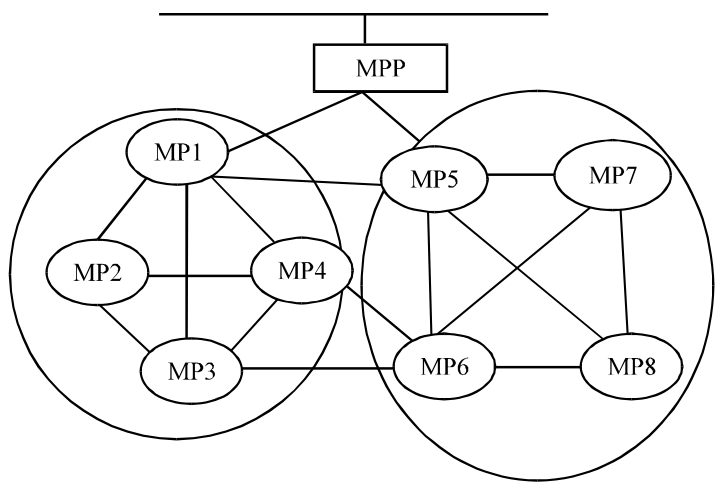

Fig. 5: Wireless mesh networks divided into cluster groups (Singh et al., 2011)

data within the cluster is too much for what the cluster gateway node can handle. This approach requires selection of new cluster gateway nodes for the new clusters formed and new routing table will be formed as well. This technique results in high delay for real time traffics while high overheads are expended on the breaking and reconstruction of clusters hence, performance is degraded.

A cluster based routing scheme was proposed by Singh et al. (2011) where routing is done by cluster heads selected to represent members of the node clusters. The proposal aimed at reducing the initial broadcast to all nodes since each cluster has one cluster head that have all information about its one hop neighbor therefore, path request only multicast to different cluster heads only. In the scheme, the researchers distribute the whole mesh network into groups as shown in Fig. 5.

MPP is saddled with the responsibility of assigning one node as a cluster head in each of the cluster group and stored the cluster head information in its own table. For every cluster head, there are two tables that were created, the first table stores neighbor cluster head information while the second table stores the cluster group member information that was assigned by the MPP. All member of a cluster stores the cluster head information in its table.

A Path Request message (PREQ) is sent to its cluster head whenever a cluster member node wants to communicate with any destination node. The cluster head check its table containing group member list to ascertain if the path or route information is there if the destination node is a member the same group with the source node, it sends path reply with path information speedily and the source node begins transmission based on the established path. However if destination node is a member of other cluster, its cluster head sends PREQ message to mesh portal then mesh portal will multicast PREQ message to all other cluster heads. 
At each of the receiving cluster heads, it checks own group table for the information of the destination node if any cluster head finds destination node in its cluster, it then send PREQ message to destination node. Destination node sends own status to its cluster head which will be used to establish path reply to mesh portal while the mesh portal relay the path to source node. The researchers claimed proposed protocol uses a smaller amount of energy and less path setup time because only one multicast and three unicast message for path discovery process were used while the later is of insignificant to infrastructure WMNs small path set up time can be of benefit to real time traffics. The researchers reasoned that fast path creation between source node and destination was due to the type of message forwarding used. Despite the feat achieved in improving network performance, maintenance of two tables by cluster heads impose high overheads also no concrete attempt was made to resolve congestion in this proposal, the believe by the researchers was that only one source node can send path request message at a time in their design whereas data traffic can aggregate at the cluster heads closer to the mesh portal whenever many source nodes transmit data traffic to one destination cluster or if the destination cluster head becomes the only path to the destination then this cluster head becomes a bottleneck for the network.

\section{PROPOSED CONGESTION AVOIDANCE AND LOAD BALANCING ROUTING FRAMEWORK}

In this study, researchers aim to propose a framework to designing a good routing which will improve the real time traffics transmission in a congested networks and balancing load along the traffic path towards the default gateway of WMNs using the IEEE802.11s. The protocol will improve the throughput in densely populated network and as well as reducing the end to end transmission delay. The method to be adopted will consider reduction on the computation and transmission overheads based on the use of clustering techniques where the cluster heads and cluster gateway nodes selection are based on node degree, node ID, cost of service and traffic intensity parameters while simple routing techniques with low overheads will be adopted. In dense traffic condition, the combinations of congestion avoidance and load balancing routing protocols will reduce the packet loss as well while also increase the quality of service of the real time application. Therefore, the proposed protocol can achieve robustness against high packet loss for a RTA in IEEE 802.11s for WMNs in a community networking scenario.
Modeling the cost of service and traffic intensity for cluster heads selection: In this study, the researchers discuss the system model and the functional requirements of the proposed model. We also looked at the model parameters and their relationship. We model the cost of service using the bandwidth usage and the average time spent by the packets sent for topology discovery. Cost of service is defined as the data packet transfer cost of the network resources used within the WMNs.

Model analysis: This cost is characterized by the function $\mathrm{f}(\mathrm{b}, \mathrm{T})$ where $\mathrm{b}$ is a measure of the bandwidth available, $\mathrm{T}$ is a measure of average service time a node takes to process or acknowledge the receipt of data traffic:

$$
\mathrm{Sc}=\mathrm{f}(\mathrm{b}, \mathrm{T})
$$

The lower the cost of service the lower the overheads, a well chosen cluster heads with lower cost of service will optimize the use of the network bandwidth. If $\mathrm{b}$ is a measure of the available bandwidth then the usage cost for such bandwidth can be derived as follows:

$$
\mathrm{b}_{\mathrm{c}}=\frac{\mathrm{b}_{\mathrm{i}}}{\mathrm{b}} \times \mathrm{d}
$$

Where:

$\mathrm{b}_{\mathrm{c}}=$ The bandwidth usage cost

$b_{i}=$ The data packet transfer per second

$\mathrm{d}=$ The distance between nodes

The distance between nodes can be calculated from Eq. 3:

$$
\mathrm{d}=\sqrt{\left(\mathrm{x}_{\mathrm{r}}-\mathrm{x}_{\mathrm{r}+1}\right)^{2}+\left(\mathrm{y}_{\mathrm{r}}-\mathrm{y}_{\mathrm{r}+1}\right)^{2}}
$$

where, $\left(\mathrm{x}_{\mathrm{r}}, \mathrm{y}_{\mathrm{r}}\right)$ and $\left(\mathrm{x}_{\mathrm{r}+1}, \mathrm{y}_{\mathrm{r}+1}\right)$ are the coordinates of nodes $\mathrm{n}_{\mathrm{r}}$ and $\mathrm{n}_{\mathrm{r}+1}$, respectively. To assess the performance of a node $n_{r}$, the notion of speed up $S$ is considered which is defined to be the ratio of the performance of a single node to the performance of many nodes in the neighborhood:

$$
\begin{gathered}
\mathrm{S}=\frac{\mathrm{T}_{\mathrm{r}}}{\mathrm{T}_{\mathrm{N}}}, \quad \mathrm{r} \in \mathrm{N} \\
\mathrm{T}_{\mathrm{N}}=\sum_{\mathrm{l}=1}^{\mathrm{N}} \mathrm{T}_{1} \\
\mathrm{~T}_{\mathrm{r}}=\mathrm{t}_{\mathrm{o}}-\mathrm{t}_{\mathrm{i}}
\end{gathered}
$$


$t_{i}$ and $t_{0}$ are the times for the receiving of data traffic and sending of acknowledgement for the received data traffic and $T_{r}$ and $T_{N}$ denote the execution time required on a node $r$ and the execution time required for all nodes in the neighborhood.

However, the speedup $\mathrm{S}$ is a function of various tasks being handled at a time $t$ hence, let the variance of speedup time be computed as:

$$
\operatorname{var}_{t} \overline{\mathrm{S}}=\frac{1}{\mathrm{~T}_{\mathrm{r}}} \sum_{\mathrm{j}}^{\mathrm{T}_{\mathrm{x}}} \mathrm{S}_{\mathrm{j}}^{2}-\left(\frac{1}{\mathrm{~T}_{\mathrm{r}}} \sum_{\mathrm{j}}^{\mathrm{T}_{\mathrm{x}}} \mathrm{S}_{\mathrm{j}}\right)^{2}
$$

Therefore, the cost of service $S_{c}$ for node $n_{r}$ can be computed thus:

$$
\begin{gathered}
S_{c}=b_{c} \times \operatorname{var}_{t} \bar{S} \\
S_{c}=\left(\frac{b_{i}}{b} \times \sqrt{\left(x_{r}-x_{r+1}\right)^{2}+\left(y_{r}-y_{r+1}\right)^{2}}\right) \times\left(\frac{1}{T_{r}} \sum_{j}^{T_{r}} S_{j}^{2}-\left(\frac{1}{T_{r}} \sum_{j}^{T_{r}} S_{j}\right)^{2}\right)
\end{gathered}
$$

Traffic intensity: For a delay sensitive product, the choice of a cluster head node that will support minimal queue delay at the cluster level is of high significance. Hence, tracking the node with minimum traffic intensity will help in determining the cluster head that can assist in reducing delay for a data packet traffic. To compute the traffic intensity $\left(\mathrm{T}_{\mathrm{i}}\right)$ the following equation applies:

$$
\mathrm{T}_{\mathrm{i}}=\frac{\mathrm{aL}}{\mathrm{R}}
$$

Where:

$\mathrm{a}=$ The average arrival rate of packets (i.e., packets $\mathrm{sec}^{-1}$ )

$\mathrm{L}=$ The average packet length (e.g. in bits)

$\mathrm{R}=$ The transmission rate (e.g., bits $\mathrm{sec}^{-1}$ )

A traffic intensity greater than one erlang means that the rate at which bits arrive exceeds the rate bits can be transmitted and queuing delay will grow without bound if the traffic intensity remain the same. If the traffic intensity is less than one erlang then the node can handle more average traffic.

The cost of service $S_{c}$ and traffic intensity $T_{i}$ will carry higher weights considering the impact of these two factors for the selection of the cluster heads that will guarantee the minimum delay and higher network performance in the clustering algorithm. Weights will be assigned here the aggregate weight will be equal to 1 .

$$
\mathrm{W}=\mathrm{W}_{\mathrm{nd}}+\mathrm{W}_{\mathrm{id}}+\mathrm{W}_{\mathrm{sc}}+\mathrm{W}_{\mathrm{ti}}=\sum_{\mathrm{f}=1}^{4} \mathrm{~W}_{\mathrm{f}}=1
$$

The cost of service metric reduces information update overheads, optimizing the use of network bandwidth.

Network nodes clustering: Having modeled the cost of service and traffic intensity, it is quite easy to determine the cluster heads which are to be saddled with the responsibility of administering the cluster and also guaranteeing inter cluster communication. The initialization mechanism is to enable each member node obtain basic information such as node ID, node distance from neighboring nodes, node degree (that is the number of neighbors to the node) and the cost of service for each node and the traffic intensity for its one-hop neighbors. These parameters are to be used with EWCA (Li et al., 2009) as the base algorithm to maintaining stable clustering configuration, minimize clustering overhead during cluster set up and for the achievement of high end to end performance. The cluster formation protocol will ensures the best candidate nodes are selected as both Cluster Heads (CHs) and intermediate Cluster Gateway Nodes (CGNs). The process of the network clustering and routing is shown in Fig. 6.

The routing phase will implement the buffer overflow and bandwidth usage control by adaptively ensure that

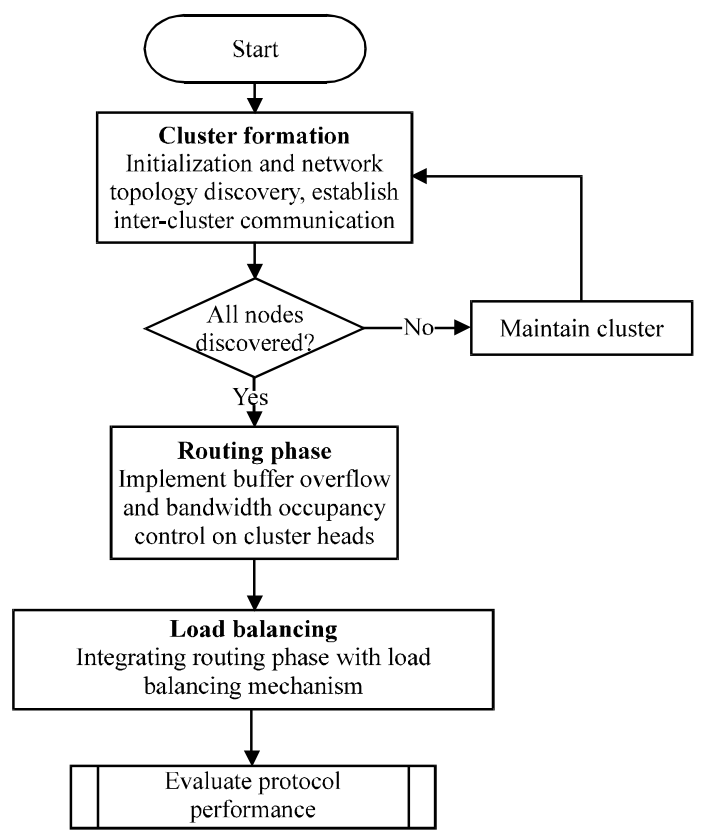

Fig. 6: Flowchart of the framework structure 
both intra and inter cluster communications suppresses the buffer from being overflowed by data packets coming from various member nodes within a cluster and from other clusters. The Congestion Avoidance Method to be employed will improve communication quality by suppressing congestion over all paths by means of combined autonomous co-operative control of the transmission rate at the intermediate nodes.

Routing model: The route discovery enables the traffic data generated by the source node to be transmitted to the destination node. In other to reduce the packet delay due to path length, the proposal will exploit shortest path algorithm. Though, shortest paths use to be highly contended paths (Jones, 2004) this may prompt serious consideration for traffic collisions. However, to ensure minimum packet drop, the congestion avoidance technique to be implemented here will take proper care of this scenario. The phase adopted preventive congestion avoidance methodology to regulate the transmission rate that is based on buffer and bandwidth state of all intermediate cluster heads or cluster gateway nodes in the network.

The Fig. 7 is framework to demonstrate the processes that each cluster head or cluster gateway node undergo before it either admit or reject any incoming data traffic packet into itself. In this design, certain assumptions are made. In the first instance, the RTAs assume to produce three types of services such as real time data, video and audio packets and that each service requires different amount of resources. No particular service is given priority over others since, they are all real time services that require prompt response, low delay and high throughput. For the purpose of simplicity, the assumptions in this design are that bandwidth and buffer are the two resources in consideration all services belonging to the same type have the same bandwidth requirements. The data traffic types are serviced when it has entered the queue based on first come first served scheduling as long as there are available resources to handle the data packets at the intermediate node. The arrival pattern of the traffic data packets is also assumed to follow Poisson distribution with different average arrival rates.

Load balancing scheme: Since, clustering helps in improving routing by reducing the size of the routing tables and by decreasing transmission overheads, the load balancing mechanism being proposed will use the clustering platform to consolidate on the gains to be achieved in the congestion avoidance module for reducing packet delay and increased throughput of the real time applications. The load balancing mechanism will be integrated with the routing protocol to establish noninterference path with a balanced traffic load leading to the default gateway to improve the network throughput and reducing end-to-end delay. The flow chart in Fig. 8 shows the load balancing process for all data packets traversing towards the gateway nodes.

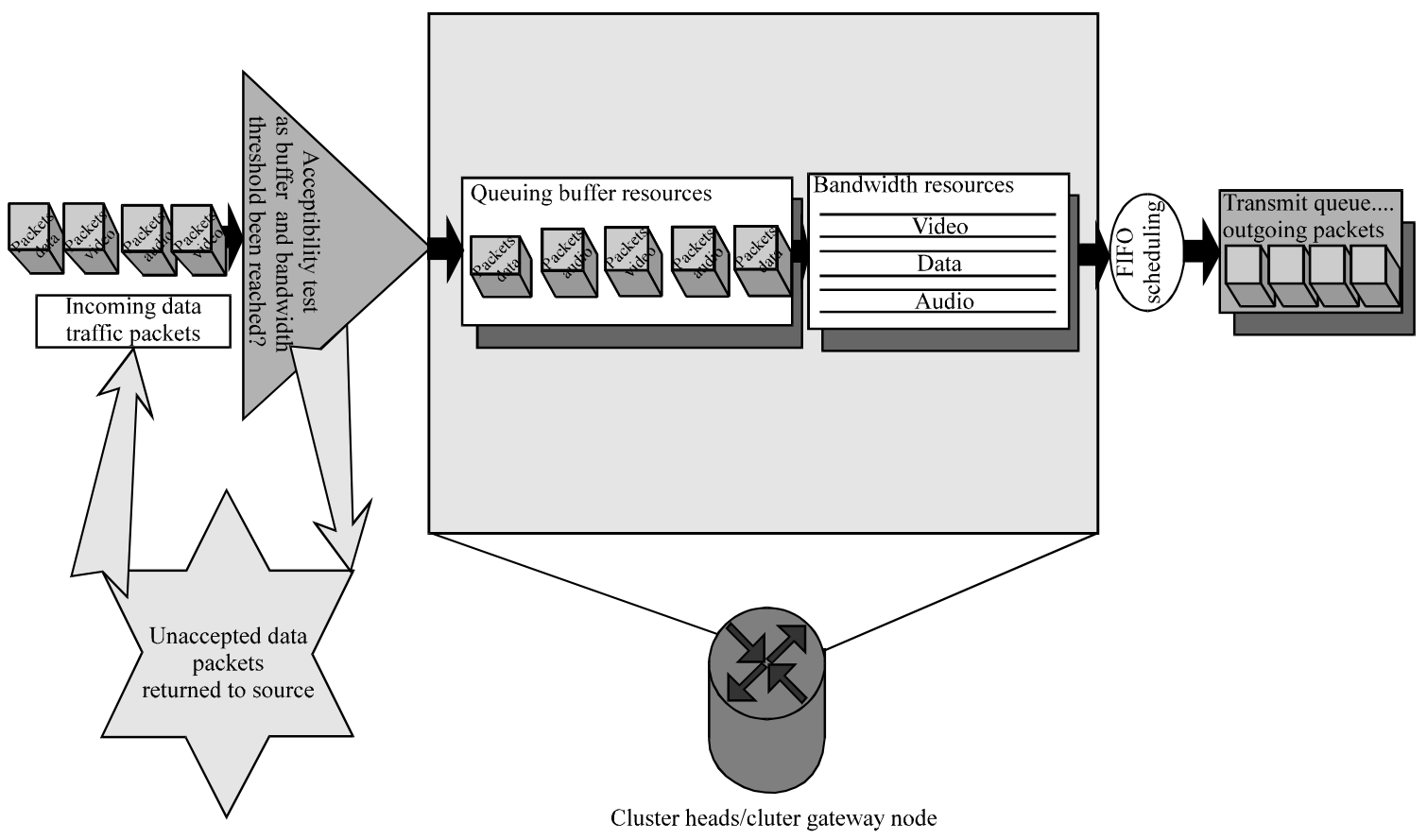

Fig. 7: Buffer occupancy and bandwidth usage control 


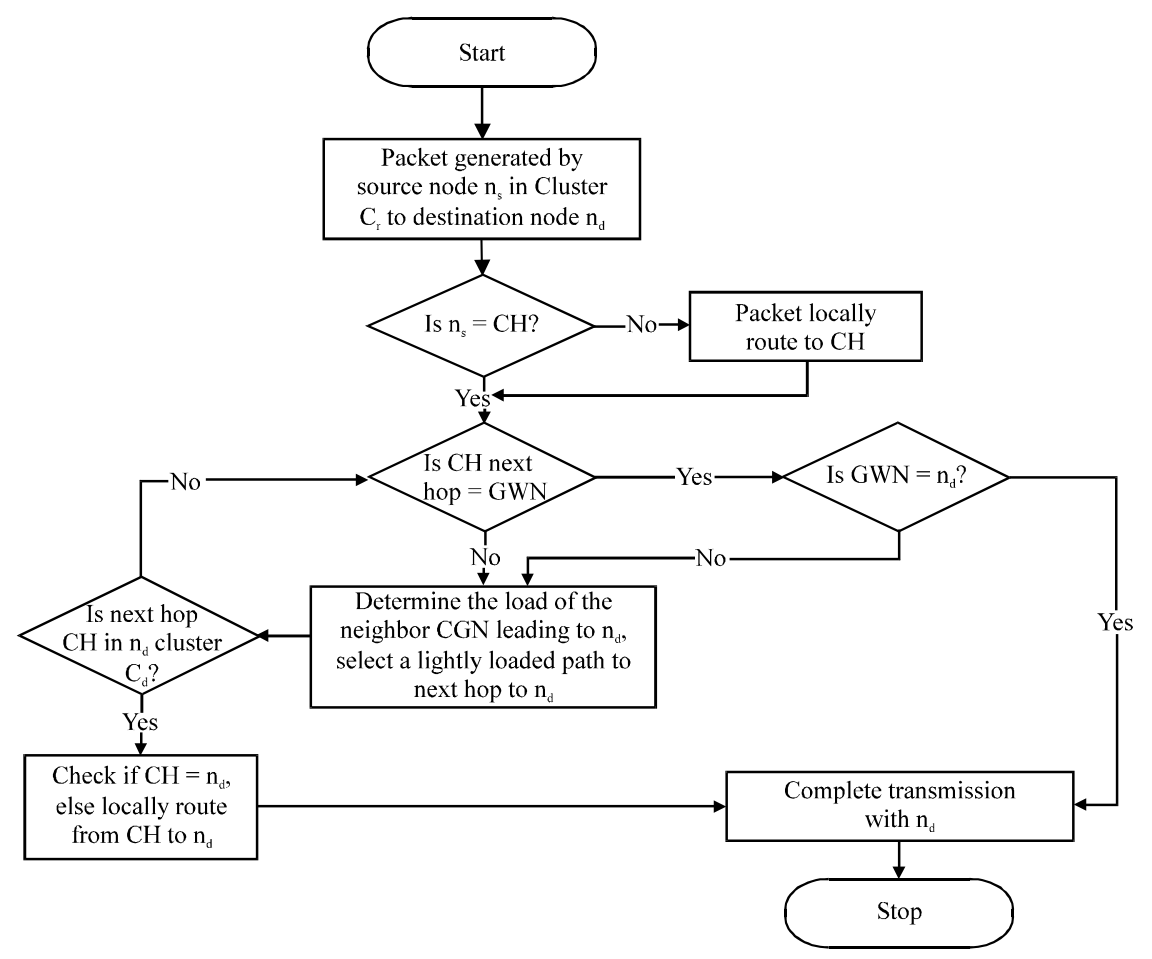

Fig. 8: Load balancing routing flow chart

The effectiveness of this approach has been proven and tested in typical WMNs (Lee and Ward, 2004) hence, this proposal will enhances this diverse routing approach for clustered infrastructure WMNs. Due to the traffic pattern where most of the data traffic are to and from designated gateway node, it is difficult to balance the load between nodes close to gateway and other nodes. This cluster based load balancing routing algorithm tries to balance load among different routing paths to the gateway in order to avoid the intra and inter flow interference while it also takes care of congestion along the traffic path.

\section{CONCLUSION}

In this study, researchers have modeled cost of service and traffic intensity parameters as part of the weighting factors for the selection of cluster heads for the purpose of clustering network nodes. Researchers also proposed a congestion avoidance and load balancing routing approach to assist in achieving solution to the identified problems in the introductory section of this study. Efforts are maintained to put up a framework which we shall use during the simulation process since, this study is an ongoing research effort to reduce buffer overflow and packet drop in an infrastructure WMNs. It is the believe that simulation results will prove this model to be better in terms of reduction in routing overheads, data packet end to end delay while also improving the network throughput and data delivery ratio for real time traffics.

\section{ACKNOWLEDGEMENT}

Researchers wish to acknowledge the Universiti Teknologi Malaysia and Federal Polytechnic Ede, Nigeria for the financial support and the provisioning of high-tech research facilities.

\section{REFERENCES}

Akyildiz, I.F. and X. Wang, 2009. Wireless Mesh Networks, Advance Texts in Communications and Networking. John Wiley and Sons Ltd., Chichester, West Sussex, England.

Akyildiz, I.F., X. Wang and W. Wang, 2005. Wireless mesh networks: A survey. Comput. Networks, 47: 445-487.

Choi, S., J. del Prado, N.S. Shankar and S. Mangold, 2003. IEEE 802.11 e contention-based channel access (EDCF) performance evaluation Int. Conf. Commun., 2: 1151-1156

Clausen, T. and P. Jacquet, 2003. Optimized link state routing protocol. Request for Comments: 3626. http://www.ietf.org/rfc/rfc3626.txt. 
Dang, H. and H. Wu, 2008. Cluster-based data transmission protocol in delay-tolerant mobile networks. Proceedings of IEEE 2008 Computer Society, September-29-October-2, 2008, Atlanta, GA pp: $529-530$.

Dang, $\mathrm{H}$. and H. Wu, 2010. Clustering and cluster-based routing protocol for delay-tolerant mobile networks. Trans. Wireless Commun., 9: 1874-1881.

Fujiwara, A., A. Yamada, L. Yang and B. Sadeghi, 2006. EDCA based congestion control for WLAN mesh networks. Proceedings of the IEEE Vehicular Technology Conference, May 7-10, 2006, Melbourne, pp: 1288-1292.

Gerla, M. and J.T.C. Tsai, 1995. Multicluster, mobile, multimedia radio network. Wireless Networks, 1: 255-265.

Hendrik, C., 1988. Routing information protocol. Routing information protocol. http://tools.ietf.org/html/ rfc1058.

Jiang, M., J. Li and Y. Tay, 1999. Cluster based routing protocol (CBRP). draft-ietf-manet-cbrp-spec-01.txt, August 1999. http://tools.ietf.org/html/draft-ietfmanet-cbrp-spec-01.

Johnson, D.B. and D.A. Maltz, 1996. Dynamic source Routing-protocols for adaptive wireless and mobile networking. IEEE J. Person. Commun., 3: 34-42.

Jones E., 2004. Multipath load balancing in multi-hop wireless mesh networks. Technical Report. University of Waterloo, Ontario, Canada.

Jun, J. and M.L. Sichitiu, 2003. The nominal capacity of wireless mesh networks. IEEE Wireless Communicat., 10: $8-14$

Kum, D.W., J.S. Park, Y.Z. Cho, B.Y. Cheon and D. Cho, 2010. Mobility-aware hybrid routing approach for wireless mesh networks. Proceedings of 2010 3rd International Conference on Advances in Mesh Networks, November 11, 2010, IEEE Computer Society, pp: 59-62.

Lee, A. and P.A.S. Ward, 2004. A study of routing algorithms in wireless mesh networks. Proceedings of Australian Telecommunication Networks and Applications Conference, December 2004, Sydney, Australia.

Li, C., Y. Wang, F. Huang and D. Yang, 2009. A novel enhanced weighted clustering algorithm for mobile networks. Proceeding of the 5th International Conferences on Wireless Communications, September 24-26, 2009, Networking and mobile Computing, Beijing, pp: 1-4.

Liu, W., C.C. Chiang, H. Wu and C. Gerla, 1997. Routing in clustered multihop, mobile wireless networks with fading channel. Proc. IEEE Sicon., 97: 197-212.
Manoj, B.S. and R.R. Rao, 2006. Load Balancing in Wireless Mesh Networks. In: Wireless Mesh Networks: Architectures, Protocols and Standards, Zhang, Y., J. Luo and H. Hu (Eds.). CRC Press, Florida, pp: 263-296.

Methley, S., 2009. Essentials of wireless mesh networking. Cambridge University Press, Edinburgh Building, Cambridge CB2 8RU, UK. ISBN: 9780521876803 , Pages: 229.

Mugaibel, H.A. and M. Othman, 2009. Review of routing protocols and its metrics for wireless mesh networks. Proceeding of the International Association of Computer Science and Infonnation TechnologySpring Conference, April 17-20, 2009 University of Putra Malaysia, Singapore, pp: 62-70.

Nandiraju, D., L. Santhanam, N. Nandiraju and D.P. Agrawal, 2006. Achieving load balancing in wireless mesh networks through multiple gateways. Proceedings of the IEEE International Conference on Mobile Adhoc and Senor Systems (MASS), Octuber 9-12, 2006, Vancouver, BC, pp: 807-812.

Perkins, C., E. Belding Royer and S.R. Das, 2003. Ad hoc on demand distance vector (AODV) routing ( $\mathrm{RFC}$ 3561). http://www.ietf.org/rfc/rfc3561 .txt.

Pinheiro, M., F. Vasques, S. Sampaio and F. Pedro, 2009. DHT-based Cluster routing protocol for IEEE 802.11s mesh networks. Proceeding of the 6 th IEEE Communication Socity Conference, Jun 22-26, 2009 , Rome, Italy, pp: 1-6.

Rahman, M.A., Saiful Azad and F. Anwa, 2009. Integrating multiple metrics to improve the performance of a routing protocol over wireless mesh networks. Proceeding of International Conference on Signal Processing Systems, May 15-17, 2009, Singapore, pp: 784-787.

Raniwala, A. and T.C. Chiueh, 2005. Architecture and algorithms for an IEEE 802.11-based multichannel wireless mesh network. IEEE INFOCOM, 3: $2223-2234$.

Schoeneich, R.O. and M. Golanski, 2007. Mesh cluster based routing protocol: Enhancing multi-hop internet access using cluster paradigm. Proceedings of the EUROCON 2007 International Conference on Computer as a Tool Warsaw, September 9-12, 2007, Warsaw, pp: 962-965.

Shahverdy, M., M. Behnami and M. Fathy, 2011. A new paradigm for load balancing in WMNs. Int. J. Comput. Networks, 3: 239-246.

Singh, M., S.G. Lee, T.W. Kit and L.J. Huy, 2011. Clusterbased routing scheme for wireless wesh networks. Proceedings of the 13th International Conference on Advance Communication Technology, February 1316, 2011, Seoul, pp: 335-338. 
Spyropoulos, T., K. Psounis and C.S. Raghavendra, 2006. Performance analysis of mobility-assisted routing. Proceedings of the 7th ACM International Symposium on Mobile ad Hoc Networking and Computing, May 22-25, 2006, Florence, Italy, pp: 49-60.

Wan, J., D. Yuan and X. Xu, 2008. A review of cluster formation mechanism for clustering routing protocols. Proceedings of 11th IEEE International Conference on Communication Technology, November 10-12, 2008, Hangzhou, pp: 611-616.
Welzl, M., 2005. Network Congestion Control-Managing Internet Traffic. 1st Edn., John Wiley and Sons Ltd., Chichester, West Sussex, England, Pages: 282.

Yi, Y. and S. Shakkottai, 2007. Hop-by-hop congestion control over a wireless multi-hop network. INFOCOM, 15: 133-144.

Zimmermann, A., D. Schaffrath, M. Wenig, A. Hannemann, M. Gunes and S. Makram, 2007. Performance evaluation of a hybrid test bed for wireless mesh networks. Proceedings of the 4th IEEE International Conference on Mobile Ad-Hoc and Sensor Systems, October 8-11, 2007, Pisa, Italy, pp: 1-6. 